\title{
A New Hysteretic Model for Magnetorheological Elastomer Base Isolator and Parameter Identification Based on Modified Artificial Fish Swarm Algorithm
}

\author{
Y. Yu, Y. Li and J. Li \\ Centre for Built Infrastructure Research, School of Civil and Environmental Engineering, Faculty of Engineering \\ and Information Technology, University of Technology, Sydney, Australia \\ E-mail: yang.yu@uts.edu.au, yancheng.li@uts.edu.au and jianchun.li@uts.edu.au
}

\begin{abstract}
-
Magnetorheological elastomer (MRE) base isolator is a new semi-active control device that has recently acquired more attention. This paper proposes a new model for MRE base isolator to portray the nonlinear hysteresis between generated force and the displacement. In this model, a hyperbolic expression is proposed to compare with the classical Bouc-Wen model, which includes internal dynamics represented by a nonlinear differential equation. For the identification of model parameters, a modified artificial fish swarm algorithm is adopted using the experimental forcedisplacement/velocity data under different testing conditions. In this algorithm, a self-adaptive method for adjusting the algorithm parameters is introduced to improve the result accuracy. Besides, the behaviours in the algorithm are simplified to descend the algorithmic complexity. Parameter identification results are included to demonstrate the accuracy of the model and the effectiveness of the identification algorithm.
\end{abstract}

Keywords-

Base isolation; Magnetorheological elastomer; Parameter identification; Artificial fish swarm

\section{Introduction}

Base isolation is the most commonly used technique for the seismic protection of civil structures [1-3]. When the earthquake occurs, the base isolation device will deflect external vibrations by isolating destructive frequency contents from transmitting into the main structure above thus keep the integrity and safety of the structure and contents therein. However, present base isolation devices are mainly passive and only effective in a narrow band of frequency range. Especially, the vulnerability for present base isolation practice during far-source and near-source earthquakes becomes a great concern. Recently, a novel adaptive base isolator based on the magnetroheological elastomer (MRE) has been put forward and realized [1]. The proposed MRE base isolator has the ability to adaptively protect base isolated structures in real-time against various types of earthquakes and outperforms the traditional seismic isolator in terms of effectiveness and functionality for the vibration protection [4-5].

MRE base isolator is a smart device with distinctive nonlinear and hysteretic behaviour, which may hinder its application in structural control. Therefore, it is of great importance that a traceable model is available before any realizable controller can be designed. So far, the research on modelling the nonlinear behaviour of MRE base isolator is relatively limited. The main model for portraying the hysteresis is the Bouc-Wen model [67]. However, due to a large number of parameters and the nonlinear equations in the model, the identification of mode parameters becomes very complicated and thus requires great computational resources. Although this problem can be solved by the direct search algorithm, it relies on the good choice of initial values to a large extent and may lead to the premature convergence. Recently, artificial fish swarm algorithm (AFSA), as a novel artificial intelligence approach, is developed for dealing with the complex problems which are difficult to be solved by other methods [8]. And it has been successfully utilized in the application of model parameter identification. Nevertheless, in virtue of the randomness of parameters and the random behaviour in basic AFSA, the algorithm has the slow convergence rate and is easy to fall into the local optimum, thus the solution accuracy is difficult to improve.

In this paper, a new computational-efficient model for MRE base isolator is proposed. This model employs a hyperbolic sine function combined with conventional viscous damping and spring stiffness to depict the hysteretic behaviour. An optimization approach based on AFSA is designed to identify the model parameters using the experimental data acquired form a practical MRE base isolator. In order to improve the result accuracy, a self-adaptive mechanism is introduced to 
update the algorithm parameters. Moreover, the behaviours in the algorithm are simplified to reduce the computational complexity in the identification process. These modifications guarantee the algorithm to have the fast convergence rate as well as the high recognition accuracy.

The reminder of the paper is organized as follows. The Bouc-Wen model and the proposed model are described in Section 2 with the statement of the optimization problem. In Section 3, the modified AFSA, as applied in identifying the proposed model parameters, is developed and its benefits are highlighted. Parameter identification results are given in Section 4 together with some analysis. Finally, Section 5 draws the conclusion

\section{System Model and Problem Statement}

The Bouc-Wen model is common-used model for describing the hysteresis response of MRE base isolators with some constraints. Accordingly, a novel model is designed in this part and an optimization problem is also presented for solving the model parameters.

\subsection{Bouc-Wen Model}

This model incorporates a Bouc-Wen component, which regenerates hysteresis loops, in parallel with a Voigt element, which depicts solid material behaviours [7]. The model can be represented by the force equation and the associated hysteretic variable, given by:

$$
\begin{gathered}
F=\alpha k_{0} x+(1-\alpha) k_{0} z+c_{0} \dot{x} \\
\dot{z}=A \dot{x}-\beta|\dot{x}||z|^{n-1} z-\gamma \dot{x}|z|^{n}
\end{gathered}
$$

Where $k_{0}$ denotes the stiffness of the spring; $c_{0}$ is the viscous coefficient indicating the damping capacity of the system; $\alpha, A, \beta, \gamma$ and $n$ are non-dimensional parameters which are responsible for the shape and size of the hysteretic loops; $z$ is the hysteretic variable that represents a function of the time history of the displacement. The Bouc-Wen model is widely used in structure engineering and MR behaviour for its mathematical ability to represent a large class of hysteretic behaviour. However, because of the incorporation of internal dynamics in regard to the hysteretic variable $z$, undesirable singularities may appear in the process of model identification.

\subsection{Proposed Model}

Compared with relatively complicated Bouc-Wen model, a simple model is presented in this work to model the nonlinear force-displacement characteristic of the MRE base isolator. A component-wise added approach is adopted which includes the viscous damper, spring stiffness and a hysteretic component. The structure of the proposed model is shown in Figure 1.

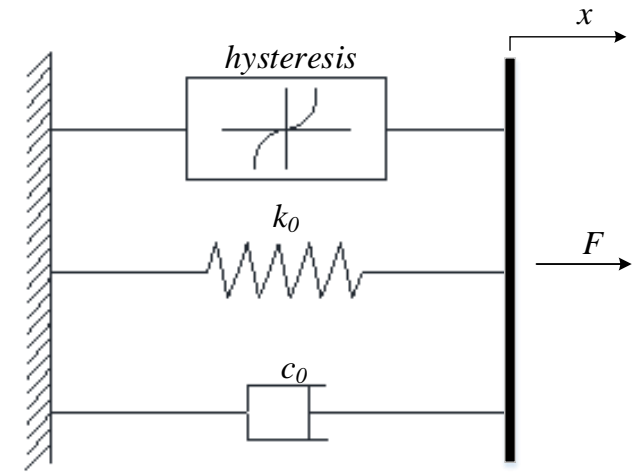

Figure 1.The proposed model

In terms of mathematical expressions, the model utilizes a hyperbolic sine function to describe the hysteresis and linear functions to describe the viscous and stiffness. The model expression is given as:

$$
\begin{gathered}
F=c_{0} \dot{x}+k_{0} x+\alpha z+F_{0} \\
z=\sinh (\beta x)
\end{gathered}
$$

Where $c_{0}$ and $k_{0}$ are the viscous and stiffness coefficients; $\alpha$ is the scale factor of the hysteresis; $z$ denotes the hysteretic variable given by the hyperbolic sine function; $\beta$ is the scale factor of the isolator displacement defining the hysteretic slope; $F_{0}$ is the isolator force offset.

\subsection{Problem Statement}

As the parameters of the proposed model are difficult to search by trials, a minimization optimization is employed to solve the problem. The critical point of the optimization is the choice of the fitness function, which has an important influence on the identification results. In this work, the fitness function $H(X)$ is defined as follows:

$$
H(X)=\frac{1}{N_{v}} \sum_{i=1}^{N_{v}}\left[F_{i}-\left(c_{0} \dot{x}_{i}+k_{0} x_{i}+\alpha \sinh \left(\beta x_{i}\right)+F_{0}\right)\right]^{2}
$$

Where $X=\left[c_{0}, k_{0}, \alpha, \beta, F_{0}\right] ; N_{v}$ is the total number of the experimental data, $F_{i}, x_{i}$ and $\dot{x}_{i}$ denote the force, displacement and velocity at the $i$ th sampling time, respectively. If the fitness value is very close to zero, 
the identification result $X$ is regarded as the optimal solution. As a consequence, the minimum optimization problem with constraints can be formulated as:

$$
\operatorname{Min}_{X} H(X) \text { s.t. } c_{0}>0, k_{0}>0
$$

Since the proposed model is nonlinear, the gradient information is not able to be formulated by explicit expressions. Hence, the traditional gradient-based optimization method could not work well. Although this problem could be solved by the direct search algorithm, however, it relies on good choice of initial values to a large extent and may trap into the local optimum. Therefore, the global search algorithm is required. In the following section, a modified artificial fish swarm algorithm is designed to identify the model parameters.

\section{Model Parameter Identification}

The process of identification for model parameters is generally accomplished by the optimization algorithm. A modified artificial fish swarm algorithm (MAFSA) is devised in this part to estimate the optimal solution of model parameters with fast convergence and high accuracy.

\subsection{Description of AFSA}

AFSA is a novel population based evolutionary computation technique inspired by the natural social behaviour of fish school and swarm intelligence [8]. In AFSA, the AF individual state can be illustrated as $X=\left(x_{1}, x_{2}, \ldots, x_{n}\right)$, where $x_{i}(i=1,2, \ldots, n)$ denotes the variable to be searched for the optimal value. The food consistence at present location can be expressed as $Y=f(X)$, where $Y$ is the fitness function. The distance between the individuals is defined as $d_{i j}=\left\|X_{i}-X_{j}\right\|$. The visual range of AF is denoted as visual, step is the maximum step length and $\delta$ is crowd factor. The essence of AFSA is to search the optimal result through the iterative algorithm. In each iteration, the AF updates its state to achieve the optimum according to the different behaviours. The following part describes the main behaviours in AFSA.

\subsubsection{Searching Behaviour}

Suppose that $X_{i}$ denotes the present state of the AF. In its sensing range, a new state $X_{j}$ is randomly chosen. If $Y_{i}>Y_{j}$, move a step in this direction; or else, choose a state randomly again and decide whether it meets the moving condition. If it still cannot meet the condition after several times (try_number), it goes a step randomly.

\subsubsection{Swarming Behaviour}

The AF with the state $X_{i}$, searches for the number $n_{f}$ of its fellows in the present visual neighborhood $\left(d_{i j}<\right.$ visual $)$ and estimates the fellows' central location $X_{c}$. Here, $Y_{c}$ represents the food concentration at the location of $X_{c}$. When $n_{f} \geq 1$, the AF estimates the central location of its fellows. If $Y_{c} / n_{f}>\delta Y_{i}$, forward a step to the fellows' center because the food concentration at the central location is high and the environmental condition is not too crowded. On the contrary, the AF performs the searching behaviour.

\subsubsection{Following Behaviour}

The AF with the state $X_{i}$, searches for the number $n_{f}$ of its fellows in the present visual neighborhood $\left(d_{i j}<\right.$ visual $)$ and find the location $X_{\max }$ of its fellow with highest food concentration. $Y_{\max }$ is denoted as the value of food concentration at the location $X_{\max }$. If $Y_{\max } / n_{f}>$ $\delta Y_{i}$, forward a step to the location $X_{\max }$ because the food concentration at the location $X_{\max }$ is high and the environmental condition is not too crowded. On the contrary, the AF performs the searching behaviour.

\subsubsection{Behaviour Selection}

The AF's behaviour is determined by its hungry degree, which is signified by energy. Here, the mean energy $\varphi$ is defined as:

$$
\varphi=\frac{1}{m} \sum_{i=1}^{m} Y_{i}
$$

If the food concentration at the location $X_{i}$ is smaller than $\varphi$, the AF executes the following behaviour to attain the food in the area with the high food concentration. If the food concentration at the location $X_{i}$ is larger than $\varphi$, the $\mathrm{AF}$ will perform the swarming behaviour to avoid the dangerous animals. If the AF does not perform the following or swarming behaviour, it will select the searching behaviour.

\subsubsection{Bulletin}

A bulletin board is set up to record the optimal individual's state and the food concentration at the present location. Update the bulletin with the better state of the AF and the final value of the board is regarded as the optimal solution of the problem. 


\subsection{Modified AFSA}

As a random searching algorithm, the AFSA has the benefits of less initial requirements, good global convergence, strong robustness, easy realization. However it still has some disadvantages in the practical operation. First of all, since some parameters such as visual and step are usually set to constants, the algorithm has the slow convergence rate in the later stage of optimization, which makes it easy to fall into the local optimum. Then, the randomness of visual and step as well as the existence of random behaviour have a strong effect on the accuracy of optimization. Furthermore, the increase of AF number requires a large storage space, which also leads to an increase of the calculation amount. Therefore, some modifications are implemented to tackle the above problems.

\subsubsection{Modification of Parameter Update}

In basic AFSA, visual and step are two important parameters with respect to the algorithm performance. When visual is set to a large value, the AF has strong global searching as well as fast convergence rate. On the contrary, the AF has strong local searching ability. Similarly, the larger the value of step is, the faster the algorithm convergence will be, even though the numerical oscillation may appear sometimes. In contrast, the smaller step is, the slower the algorithm convergence will be, but the solution has the higher accuracy.

Based on the above analysis, in order to improve the global searching ability and the convergence rate of the AFSA, the larger visual and step are selected to make the AF search in a larger scope in the initial phase of the optimization. With the process of iteration, visual and step decline gradually so that the AF can carry out the local search in the adjacent domain of the optimal solution, which is aimed to increase the algorithm accuracy. In this paper, an inertia weight $\omega$ based on the exponential function is designed to dynamically update visual and step. The update equations are given as:

$$
\left\{\begin{array}{l}
\omega=\exp \left[-20 \cdot\left(\text { iter } / T_{\max }\right)^{p}\right] \\
\text { visual }=\text { visual }_{\min }+\omega \cdot \text { visual } \\
\text { step }=\text { step }_{\min }+\omega \cdot \text { step }
\end{array}\right.
$$

Where iter is the current iteration number; $T_{\max }$ is the maximal iteration number; $p$ is an integer to control the change rate of parameters with the range [1, 30]; visual $_{\text {min }}$ and step $_{\text {min }}$ denote the minimal visual range and step length, respectively. Generally, both visual and step are the piecewise functions, which keep the maximal values in the early stage, descend gradually in the intermediate stage and retain the minimal values in the later stage. Figure 2 shows the inertia weight curves for different values of $p$.

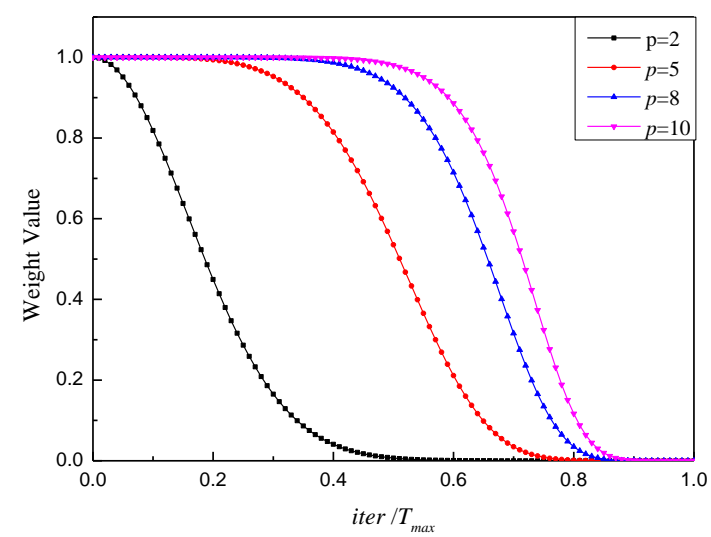

Figure 2. The weight value

\subsubsection{Modification of AF's Behaviour}

In the conventional searching behaviour, the $\mathrm{AF}$ randomly selects a state and forwards a step to this state if it is superior to the present state. This operation would slower the search speed of the AFSA when the dimension of the AF is relatively higher. Furthermore, in the swarming and following behaviours, distances between the $\mathrm{AF}$ and others in its visual range are calculated to estimate the central location and optimal location, respectively. However, with the increase of the number of the AF, the calculation amount of the algorithm will become increasingly large, which may result in the long running time.

Aiming at the above drawbacks, some modifications for the AF behaviours have been conducted to improve the algorithm performance. In order to enhance the algorithm search speed, the AF directly moves to the optimal location in its visual range if it meets the condition of the searching behaviour. Besides, in order to reduce the computational complexity, the central and optimal locations in AF's visual range are replaced by the responding ones in the whole swarm, respectively. This modification avoids the calculation for the distances between AF and others in its visual range and greatly shortens the running time.

\subsection{Steps of Modified AFSA}

In this part, the MAFSA is utilized to identify the parameters of the MRE base isolator model. The detailed process of parameter identification consists of the following steps:

Step1. Initialize the optimization problem and 
algorithm parameters: the problem is defined as Minimize $H(X)$ subject to $X_{l} \leq X \leq X_{u}$, where $X=\left[k_{0}\right.$, $\left.c_{0}, \alpha, \beta, F_{0}\right], X_{l}$ and $X_{u}$ denote the lower and upper bounds of the variable $X$. the algorithm parameters include the number of the AF $N$, visual min $_{\text {min }}$ step $_{\text {min }}, p, \delta$, try_number and $T_{\text {max }}$.

Step 2. Initialize the AF swarm: the initial locations of all the AF are generated randomly within the available range. Then set the initial iteration number iter $=0$.

Step 3. Evaluate the fitness value: calculate and compare the food concentration of initial AF, then record and conserve the biggest one on the bulletin board.

Step 4. Update the values of visual and step according to equation (8).

Step 5. Behaviour operation: the AF performs the searching behaviour, swarming behaviour or following behaviour according to its hungry degree.

Step 6. Check the AF self-state and information on the bulletin board: if the fitness value is superior to that on the board, update the board. Or else, it remains unchanged.

Step 7. Check the termination criterion. If the iteration number is equal to the maximum iterations or the solution for the optimization problem is equal to the target value, perform Step 8. Or else, iter $=$ iter +1 and go to Step 4.

Step 8. Terminate the algorithm: output the optimal solution and responding the fitness value of the AF (that is the optimal model parameters and food concentration on the board).

In conclusion, the process of the MAFSA is illustrated in Figure 3.

\section{Identification Results and Analysis}

In order to validate the effective of the new model and the availability of the proposed MAFSA, MRE base isolator parameter identification processes will be carried out and compared in the context of model accuracy and algorithm performance.

\subsection{Experiment Setup}

Several groups of experimental data are acquired from a MRE base isolator and fed into the MAFSA to identify the parameters of the proposed new model. The isolator is excited by a sinusoidal displacement in several test cases with different excitation displacements and magnetization currents supplied to the MRE base isolator. The driving frequency is set as $1 \mathrm{~Hz}$, the current ranges from $0 \mathrm{~A}$ to $3 \mathrm{~A}$ whilst the displacement is varied from $2 \mathrm{~mm}$ to $8 \mathrm{~mm}$, respectively. The excitation displacement has 3 settings and the current contains 4 entries. Therefore, a total of $3 \times 4=12$ experimental data sets are made up, which can be summarized in Table 1 . The parameters of MAFSA are set as follows: $N=50$, try_number $=50, \quad \delta=0.6, \quad T_{\max }=300, \quad$ visual $_{\min }=0.002$, step $_{\min }=0.0003$ and $p=5$. Moreover, the identification is conducted using both the Bouc-Wen model and the proposed model while the algorithms adopted contain MAFSA and other conventional ones.

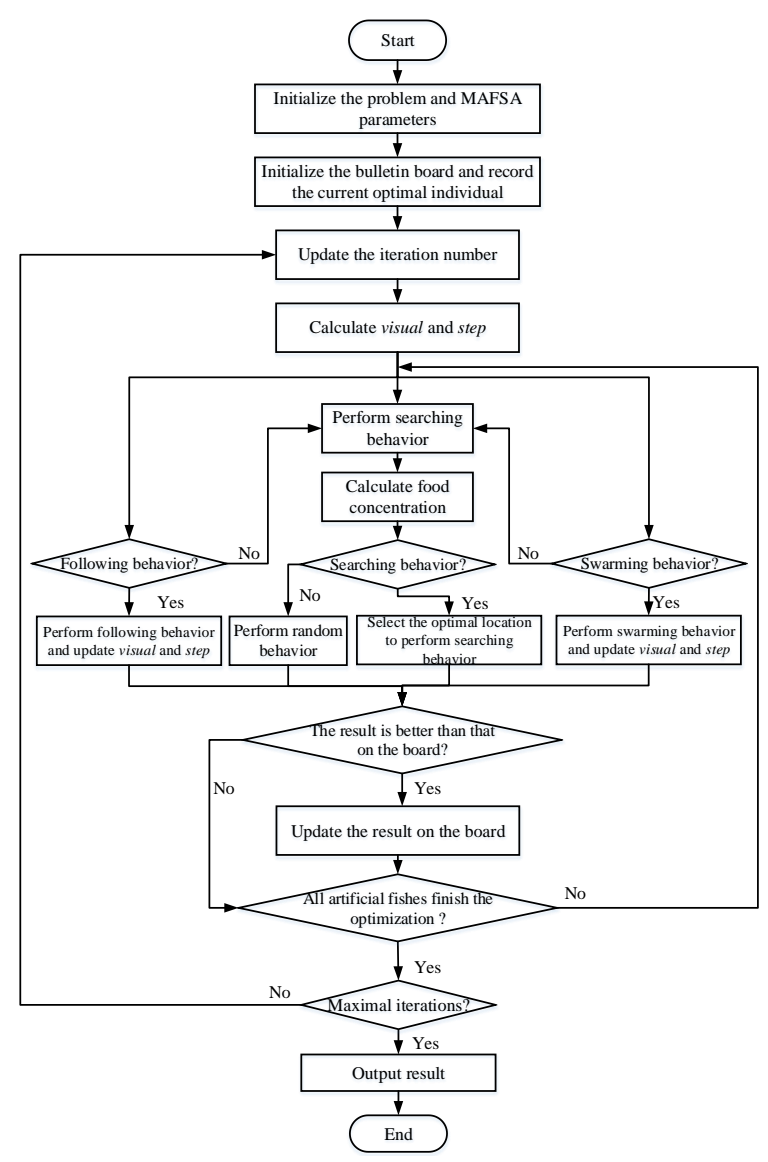

Figure 3. The process of MAFSA

Table 1. Experimental conditions

\begin{tabular}{ccccccc}
\hline Frequency $(\mathrm{Hz})$ & 1 & 1 & 1 & 1 & 1 & 1 \\
Current $(\mathrm{A})$ & 0 & 0 & 0 & 1 & 1 & 1 \\
Displacement $(\mathrm{mm})$ & 2 & 4 & 8 & 2 & 4 & 8 \\
\hline Frequency $(\mathrm{Hz})$ & 1 & 1 & 1 & 1 & 1 & 1 \\
Current $(\mathrm{A})$ & 2 & 2 & 2 & 3 & 3 & 3 \\
Displacement $(\mathrm{mm})$ & 2 & 4 & 8 & 2 & 4 & 8 \\
\hline
\end{tabular}

\subsection{Identification results}

To validate the effectiveness of the new model to predict the performance of the MRE base isolator, a group of data $(1 \mathrm{~Hz}, 0 \mathrm{~A}$ current and $2 \mathrm{~mm}$ amplitude) 
are used for parameter identification. The optimal results are given in Table 2. Figures 4 and 5 show the tracking process in a sampling cycle and relative errors between the experimental and predicted forces, respectively. It can be seen that the relative error is within $\pm 6 \%$, which is acceptable in the modelling study. Figures 6 and 7 demonstrate the force-displacement responses and the nonlinear relationship between force and velocity. It is clearly shown that the estimated forces resemble the practical testing ones very well, especially in the regions where the strain stiffening is evident.

In order to further verify the ability of the model for depicting the hysteretic behaviours of the MRE base isolator, more groups of comparisons between the practical testing and predicted data corresponding to different loading conditions are conducted. Figures 8 and 9 reveal that the estimated data is well fitted to the experimental data under the conditions of $3 \mathrm{~A}, 1 \mathrm{~Hz}$ frequency for $2 \mathrm{~mm}, 4 \mathrm{~mm}$ and $8 \mathrm{~mm}$ amplitudes respectively. It is clearly seen that experimentally measured responses are reasonably modelled. The measured force-displacement pairs shown in Figure 10 are acquired by loading the isolator with a $1 \mathrm{~Hz}$ sinusoid and a $4 \mathrm{~mm}$ amplitude at three current levels, 0A, 1A, $2 \mathrm{~A}$ and $3 \mathrm{~A}$, respectively. The four group comparisons validate the capacity of the model to portray the increasing nonlinearity of the hysteretic loops with the increasing currents. In particular, in each hysteresis loop, the estimated data resembles the unique behaviour of straining hardening perfectly.

Table 2. Parameter values of the proposed model

\begin{tabular}{cccccc}
\hline Parameter & $c_{0}$ & $k_{0}$ & $\alpha$ & $\beta$ & $F_{0}$ \\
\hline Value & 0.354 & 3.572 & 4.579 & 0.302 & 0.001 \\
\hline
\end{tabular}

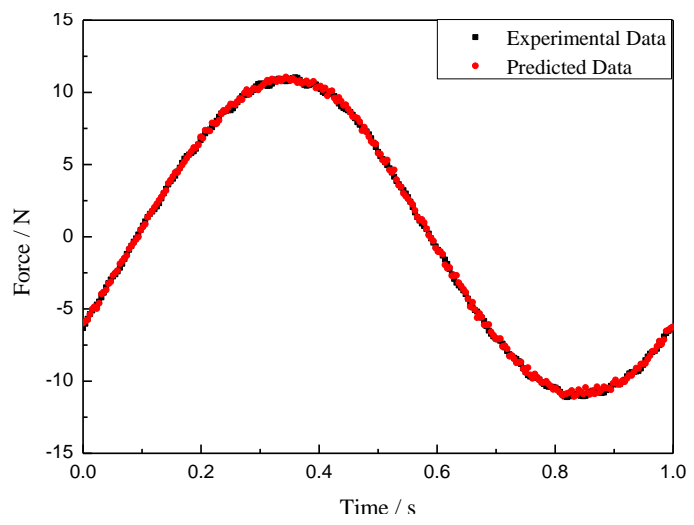

Figure 4. Force vs. time

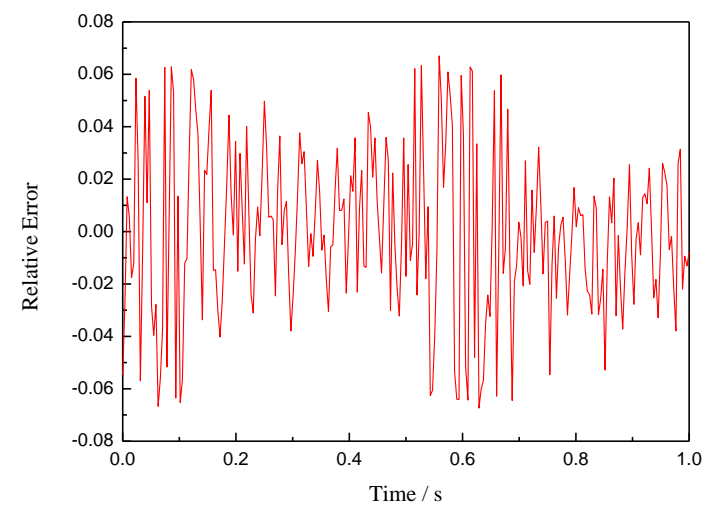

Figure 5. Relative error vs. time

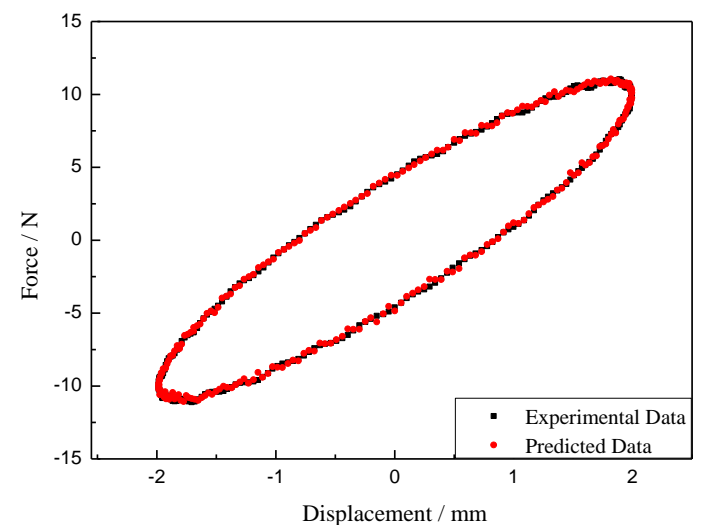

Figure 6. Force-displacement response

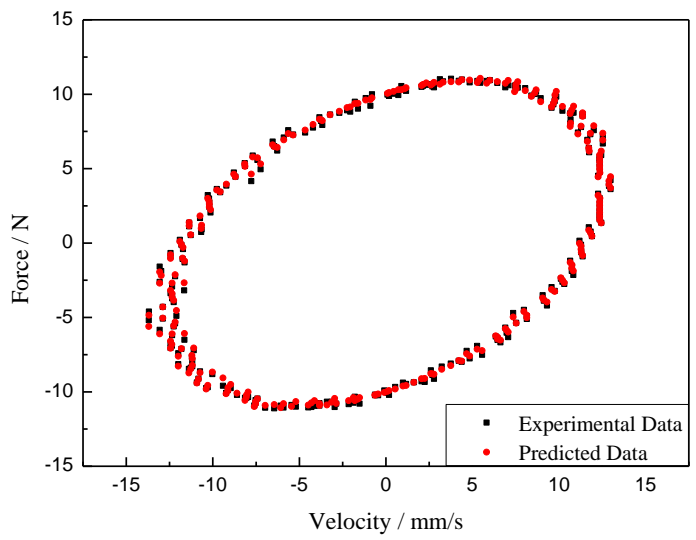

Figure 7. Force-velocity response

To evaluate the accuracy of the proposed model, a root-mean-square (RMS) is adopted as the assessment criteria given by:

$$
e_{r m s}=\sqrt{\frac{1}{N_{v}} \sum_{i=1}^{N_{v}}\left(F_{i}^{p r e}-F_{i}^{e x p}\right)^{2}}
$$




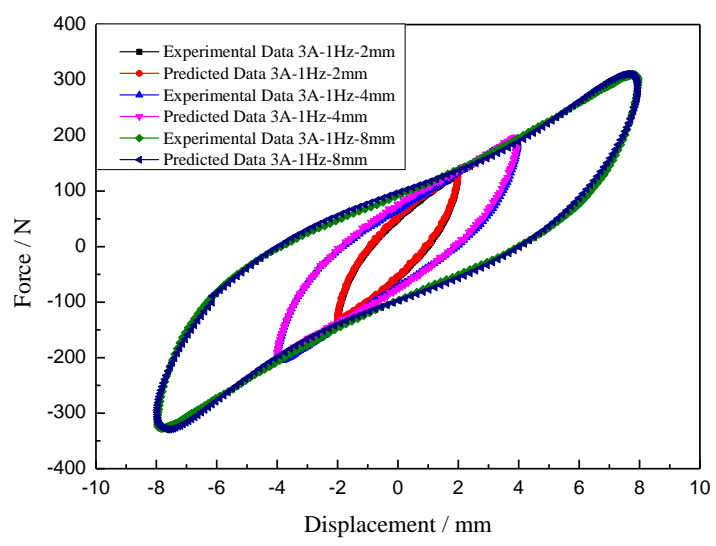

Figure 8. Force-displacement response under different loading amplitudes

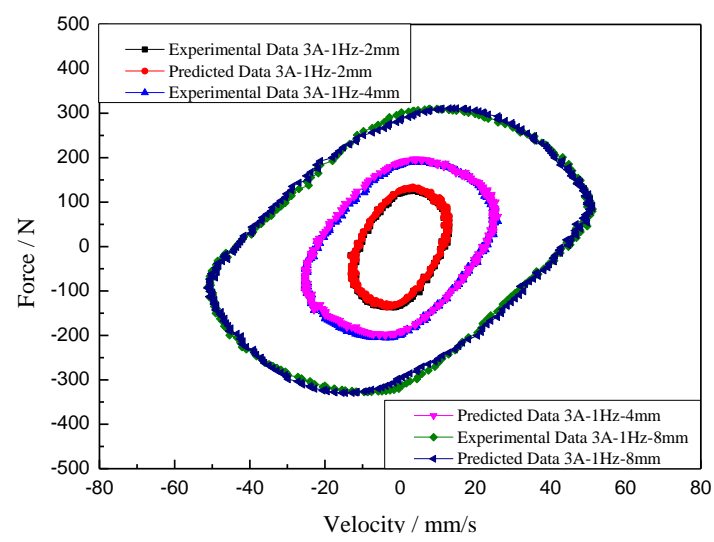

Figure 9. Force-velocity response under different loading amplitudes

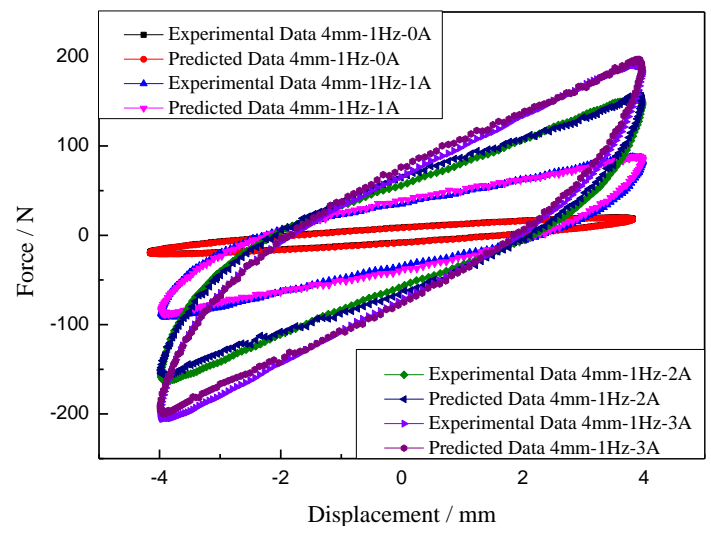

Figure 10. Force-displacement response under different loading currents

In the meantime, the conventional Bouc-Wen model is also used to compare with the proposed one for model error analysis. Figure 11 shows the comparison result. It is evidently seen that because of the higher degree of nonlinearity in the Bouc-Wen model, a bigger RMS error is shown from the predicted force from the model. For another thing, the errors in the proposed model are generally less than that of the Bouc-Wen model.

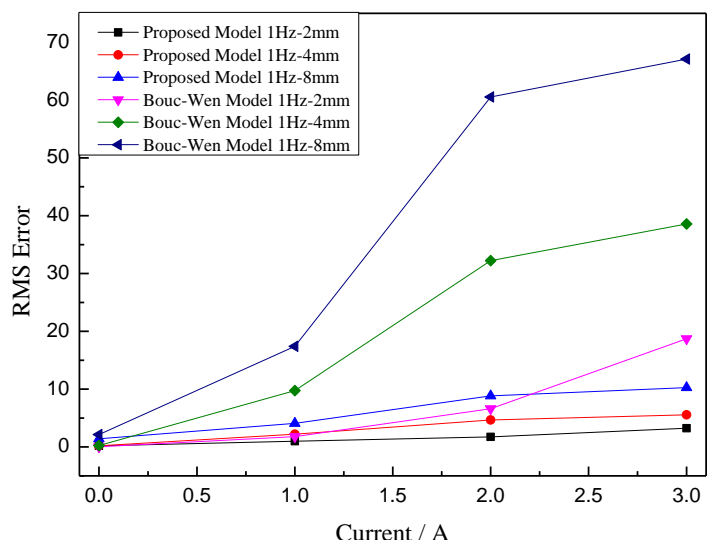

Figure 11. RMS error for both Bouc-Wen model and proposed model

\subsection{Identification Algorithm Analysis}

The efficiency of the identification process is generally associated with the model complexity and the parameter values of the optimization algorithm. Here, the model is determined and the influence of visual and step on the optimization process has been illustrated in 3.2. So in this part, the AF population $N$, as one of most important parameters, is studied for its effect on the algorithm perforce. Figure 12 describes the convergence of the MAFSA with different population number over 300 iterations under the loading condition of $1 \mathrm{~A}$ current, $1 \mathrm{~Hz}$ sinusoid and $4 \mathrm{~mm}$ amplitude, and the optimal fitness value and calculation time are listed in Table 2 . It is obvious that with the increase of the AF number, the MAFSA has the higher identification accuracy as well as more calculation time. However, when the population number exceeds 50 , the fitness value varies very slightly. Thus, the selection of 50 as the AF population is reasonable.

So as to demonstrate the superiority of the MAFSA, two conventional optimization algorithms are adopted for performance comparison: (a) AFSA with fixed visual and step; (b) Particle Swarm Optimization (PSO) [9]; (c) MAFSA. For the purpose of fair assessment, all the parameter values in AFSA are similar to that of MAFSA, except visual and step. Figure 13 shows the comparison result. It is shown that although the AFSA has the fastest convergence among three algorithms, it results in the premature convergence. Compared with the PSO, MAFSA arrives at its optimum more quickly and has higher identification accuracy. 


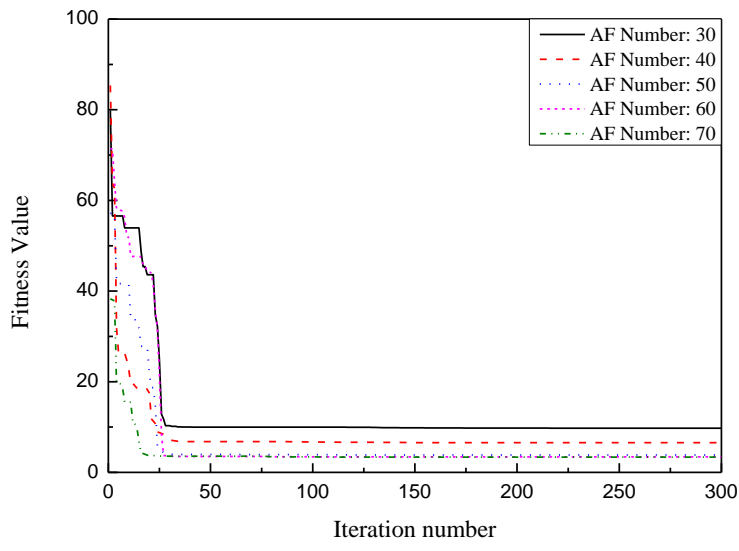

Figure 12. Convergence of MAFSA with different AF population number

Table 3. Parameter values of the proposed model

\begin{tabular}{ccc}
\hline AF number & Fitness value & Calculation time (s) \\
\hline 30 & 9.754 & 156.457 \\
40 & 6.551 & 192.214 \\
50 & 3.842 & 239.571 \\
60 & 3.426 & 275.786 \\
70 & 3.360 & 323.143 \\
\hline
\end{tabular}

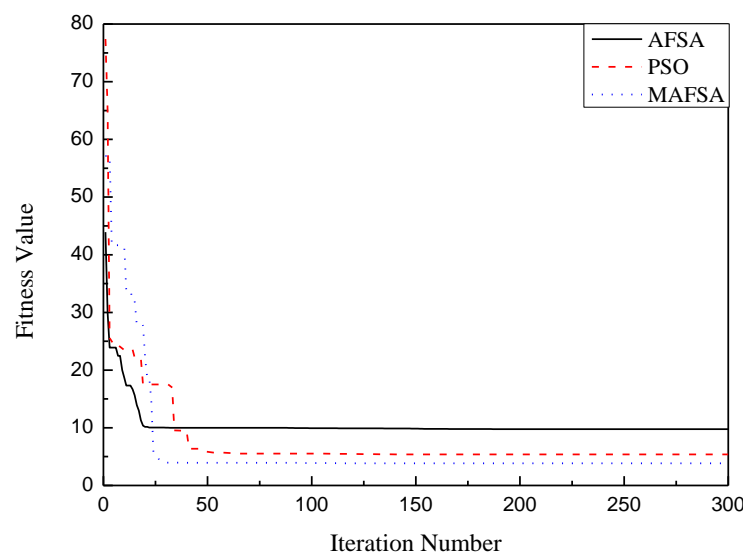

Figure 13. Algorithm performance comparison

\section{Conclusion}

This paper has presented a new model for MRE base isolators and an efficient optimization algorithm based on AFSA for model parameter identification. The new model adopts the hyperbolic sine function to describe the hysteretic relationship between the isolator force and displacement, and requires as smallest as five parameters in contrast with eight parameters of the Bouc-Wen model. An efficient optimization algorithm based on AFSA is also designed for the model parameter identification. In order to improve the convergence rate and reduce the calculation amount of the identification process, a self-adaptive parameter update approach is introduced and some behaviours in basic AFAS are also simplified. Experimental data from a practical MRE base isolator are utilized for modelling verification. The results obtained by the new model have shown highly satisfactory coincidence with the experimental data, and also the effectiveness of the proposed identification algorithm.

\section{References}

[1] Li Y., Li J., Tian T. and Li W. A highly adjustable magnetorheological elastomer base isolator for real-time adaptive control. Smart Material Structures, 22(9): 095020, 2013.

[2] Kelly J. M. Base isolation: linear theory and design. Earthquake Spectra, 6(2): 223-244, 1990.

[3] Li J., Li Y., Li W. and Samali B. Development of adaptive seismic isolators for ultimate seismic protection of civil structures. In Proceedings of SPIE 8692, Sensors and Smart Structures Technologies for Civil, Mechanical, and Aerospace Systems, 86920H, San Diego, USA, 2013.

[4] Li Y., Li J., Li W. and Samali B. Development and characterization of a magnetorheological elastomer based adaptive seismic isolator. Smart Material Structures, 22(3): 035005, 2013.

[5] Popp K., Zhang X., Li W. and Kosasih P. MRE properties under shear and squeeze modes and applications. Journal of Physics: Conference Series, 149(1): 012095, 2009.

[6] Kwok N. M., Ha Q. P., Nguyen M. T., Li J. and Samali B. Bouc-Wen model parameter identification for a MR fluid damper using computationally efficient GA. ISA Transactions, 46(2): 167-179, 2007.

[7] Yang J., Du H., Li W., Li Y., Li J., Sun S. and Deng H. Experimental study and modeling of a novel magnetorheological elastomer isolator. Smart Material Structures, 22(11): 117001, 2013.

[8] Huang Z. and Chen Y. An improved artificial fish swarm algorithm based on hybrid behaviour selection. International Journal of Control and Automation, 6(5): 103-116, 2013.

[9] Zhang H., Li X., Li H. and Huang F. Particle swarm optimization-based schemes for resourceconstrained project scheduling. Automation in Construction, 14(3): 393-404, 2005. 